\title{
O que facilita e o que dificulta uma consulta
}

Rizério Salgado*

\section{RESUMO}

As consultas consideradas «difíceis» são frequentes em medicina geral e familiar, perturbam a prestação de cuidados e são fonte de insatisfação para o médico e para o paciente. Neste artigo são analisadas características dos dois intervenientes e das circunstâncias que podem contribuir para a ocorrência de uma consulta difícil, sendo sugeridas estratégias para melhorar a comunicação médico-paciente e a gestão deste tipo de consultas.

Palavras-chave: Pacientes Difíceis; Consultas Difíceis; Comunicação Médico-Paciente.

\section{INTRODUÇÃO}

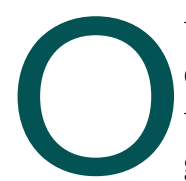

trabalho médico é em grande parte feito de encontros com os pacientes, deles resultando, na maioria das vezes, uma sensação gratificante para ambas as partes. No entanto, uma porção desses encontros provoca no clínico ansiedade, incómodo, sensações depressivas, frustração, fúria ou outros sentimentos negativos. Tais acontecimentos podem ser potenciados pelo médico, pelo paciente ou por outras circunstâncias.

Há indicações de que a prevalência de consultas consideradas difíceis é de cerca de $15 \%^{1}$ e de que, independentemente do que as origina, delas resulta menor concentração na efectividade de cuidados, desperdício de energia pelo médico, protestos de pacientes e funcionários e manutenção dos problemas de saúde do paciente. $^{2}$

Estes pacientes criam no médico uma ansiedade antecipada, têm mais consultas do que a maioria, apresentam inúmeros problemas crónicos e agudos, são sujeitos a mais prescrições, efectuam mais exames e são referenciados com maior frequência. ${ }^{3}$ Têm ainda uma maior probabilidade de apresentarem perturbações depressivas ou ansiosas, expectativas irrealistas, baixa capacidade funcional e menor satisfação. ${ }^{1}$

Steinmetz e Tabenkin (2001) procuraram caracterizar a percepção que os médicos tinham de um «paciente difícil» através de entrevistas a médicos de família ex-

*Assistente de Medicina Geral e Familiar

USF S. Julião

Centro de Saúde de Oeiras perientes, ${ }^{4}$ concluindo que os pacientes sentidos como difíceis não eram os que apresentavam mais patologias severas ou complicadas, mas aqueles cuja atitude era rude, exigente, agressiva, que visava ganhos secundários, com doenças psicossomáticas ou alterações psiquiátricas.

\section{O QUE DEFINE UM PACIENTE DIFÍCIL?}

Elder, Ricer e Tobias (2006) efectuaram entrevistas a 102 «prestigiados médicos de família $»^{5}$ perguntando-lhes como descreviam os seus «pacientes difíceis». Nas respostas obtidas sobre as características dos pacientes foram, principalmente, mencionadas as atitudes exigentes e manipuladoras e aquelas que contribuíam para a manutenção da própria doença, enquanto as respostas referentes aos problemas médicos incidiram, predominantemente, sobre os que se apresentavam com queixas múltiplas, com pedidos de específicos de substâncias, com dor crónica ou as personalidades borderline.

Um considerável número de pacientes com quem é difícil lidar apresenta critérios de diagnóstico para perturbação de personalidade. ${ }^{6}$ Os pacientes com traços de perturbação de personalidade, mesmo em níveis subclínicos, podem causar problemas na interacção médico-paciente e tendem a ser excessivamente dependentes, exigentes, manipuladores, teimosos ou autodestrutivos, recusando os tratamentos. ${ }^{7}$

Apesar de todos os avanços tecnológicos, ${ }^{8}$ as relações entre o médico e o paciente são o centro da medicina e do trabalho médico. Na verdade, o momento da 
consulta é um momento de interacção entre duas pessoas que transportam emoções e de onde resultam novos sentimentos.

Na tentativa de ajudar os médicos a melhorar a qualidade das suas interacções com pacientes mais «complicados», alguns autores tentaram definir as características dos «pacientes difíceis». Ao mesmo tempo, outros centraram-se sobre as características do médico. Como resultado destas reflexões, a maioria dos médicos reconhece agora que o centro do problema está no encontro e não apenas nos pacientes. ${ }^{9}$ As emoções são uma constante na consulta: as do paciente, as do médico e as que resultam das suas interacções. O desafio está no seu reconhecimento, na gestão que delas fazemos e na forma com as poderemos utilizar como um instrumento útil para a prestação de cuidados.

No contexto clínico, a percepção das emoções pelo médico é essencial para a compreensão dos pacientes e dos seus problemas, sublinhando a importância da relação na medicina geral. ${ }^{10}$

\section{FACTORES QUE CONTRIBUEM PARA QUE UMA CONSULTA SE TORNE DIFÍCIL}

\section{O papel do médico}

As atitudes e os comportamentos dos médicos podem, de facto, contribuir para tornar uma consulta num acto penoso. Os clínicos com grande sobrecarga de trabalho apresentam uma maior probabilidade de ter mais pacientes que consideram difíceis. ${ }^{11}$ Aqueles médicos para quem os diagnósticos objectivos constituem uma grande necessidade terão uma maior probabilidade de relatar a presença deste tipo de pacientes, particularmente relativamente aos que apresentam sintomatologia vaga ou múltipla, que voltam sempre com uma má resposta aos tratamentos, com queixas físicas inespecíficas, desorganizados ou incumpridores. ${ }^{12-14}$

Numa sistematização das propostas de Adams e Murray, foram sugeridas algumas características dos médicos que facilitam a ocorrência de consultas difíceis: ${ }^{15}$

- Médicos zangados ou defensivos - os médicos em burnout ou insatisfeitos têm maior probabilidade de reagir negativamente aos pacientes.

- Médicos cansados ou assoberbados - os médicos têm frequentemente uma carga horária excessiva podendo da fadiga resultar menor atenção ou interesse no paciente, e como tal mais erros médicos.
- Médicos dogmáticos e arrogantes-as convicções do profissional podem não deixar espaço para o paciente se exprimir ou tomar as suas próprias decisões, $o$ que é imprescindível numa relação médico-paciente saudável.

Os médicos com menor sensibilidade e aptidão psicossocial sentem com maior frequência que os seus encontros com os pacientes são difíceis. ${ }^{16} \mathrm{O}$ médico mais experiente tem menos probabilidade de sentir um doente como «difícil» e aceita melhor a grande diversidade de comportamentos dos seus pacientes. ${ }^{4}$

\section{O médico durante o internato}

A condição de Médico Interno é propícia a algumas dificuldades adicionais. Em geral, trata-se de um médico mais jovem, com menos experiência, mais inseguro e menos treinado em lidar com situações sociais ou emocionalmente complexas. Principalmente, no princípio do Internato Complementar, as circunstâncias formativas confrontam os jovens médicos com pacientes que podem ver frustrada a expectativa de serem consultados pelo «seu médico». Sentindo-se desconfortáveis, podem exibir desconfiança, distância, inibição, muitas vezes levando o clínico a sentir-se incompetente, pensando «que não era ele que devia estar ali». Outras vezes, pelo contrário, é uma oportunidade para se contar a «história toda» que o outro médico já conhece, de ter um tempo de antena que de outra forma não conseguiria ou para se tentar manipular o novo interlocutor.

O facto de utilizar uma lista já formada, que pertence a um outro médico e cujo modo de efectuar a consulta possui dinâmica e regras próprias, pode não ser facilitador. Um «orientando», naturalmente, tenderá a respeitar a organização da consulta, as estratégias face a determinado paciente ou as opções terapêuticas, mesmo que não sejam as suas escolhas. Embora se espere que esta contradição possa constituir um desafio para o diálogo, com ganhos formativos, ela também pode originar grandes tensões no exercício profissional e na relação Orientador-Interno-Paciente. Desta forma, na mesma lista de utentes, os pacientes considerados «difíceis» podem não coincidir e variar no tipo e na quantidade, em função das características do médico e do momento formativo.

A pressão formativa, as avaliações periódicas e a carga de trabalho a que um interno pode estar sujeito, não 
são aspectos emocionalmente facilitadores da consulta. Por outro lado, um «paciente difícil» pode constituir uma excelente oportunidade formativa. Por vezes, porém, o seu encaminhamento para o interno apenas se traduz na oportunidade para o orientador não ter que «aturar um doente chato».

É fundamental que o orientador saiba fazer a ponte entre o paciente e o interno, tendo consciência das dificuldades, facilitando a relação, transmitindo a noção de equipa, mantendo a disponibilidade e, por vezes, assistindo ao fim da consulta. Num contacto posterior, pode ser importante mostrar ao paciente que há uma comunicação contínua entre o orientador e o interno.

Ser orientador pressupõe disponibilidade, mas também uma maior exposição da sua própria prática clínica. O diálogo entre os dois médicos, a reflexão conjunta e a mútua capacidade de aprendizagem constante, podem minimizar as dificuldades com os pacientes e aumentar a satisfação profissional de ambos.

\section{A incerteza}

O sentimento de incerteza é inevitável na prática médica e de forma mais acentuada nos cuidados de saúde primários, onde muitas decisões não são baseadas na evidência, onde não há uma matemática clínica definida para todas as áreas, onde existem situações complexas, fronteiras indefinidas, quadros inespecíficos e a gestão de um sem número de idiossincrasias. «A incerteza insinua-se em toda a medicina e temos que saber lidar com a dor e a culpa que ela transporta» (Colin Guthrie).

O médico pode ter dificuldade em mostrar as suas dúvidas por temer que tal vá defraudar o paciente e, numa medicina centrada no médico, este terá mais probabilidades de se sentir incompetente e com baixa auto-estima. Na verdade, os resultados incertos, a ameaça da morte ou de queixas, os problemas insolúveis, as somatizações ou a dependência do paciente num modelo de consulta centrado no médico, podem predispor a mais situações de incerteza.

Mais do que temer a incerteza, aceitá-la como parte do processo clínico é o primeiro passo no desenvolvimento de estratégias com vista a minimizar o desconforto que lhe está associado. Várias pistas têm sido sugeridas, como ter disponível no gabinete um sistema de informação e de apoio à decisão clínica, adquirir capa- cidade para adoptar atitudes adequadas em termos de inteligência emocional, desenvolver auto-reflexão e adquirir auto-consciência, construir capacidade de resiliência através de apoio emocional ou de estilos de vida saudáveis e partilhar as dúvidas com os colegas.

Na consulta, a verbalização da incerteza, sistematizando os vários dados em jogo e as expectativas e emoções do paciente, ajudarão ao processo de negociação de decisões, de partilha de responsabilidades e do risco. Neste modelo, o paciente certamente sentirá maior compreensão e empenhamento por parte do médico na resolução do puzzle clínico.

Dizer a um paciente que não se tem uma resposta imediata, mas que se irá estudar o seu caso ou falar com outros colegas, trará mais benefícios à relação médico-paciente do que a resposta impulsiva de um médico mais rígido.

\section{Factores circunstanciais}

Nas últimas décadas, a relação dos pacientes com os cuidados de saúde alterou-se substancialmente. Existindo um maior número e diversidade de fontes de informação facilmente acessíveis, os médicos passaram a ser mais confrontados com dúvidas, desconfianças e transmissão de outras opiniões. Às velhas crenças, juntaram-se algumas outras, como os poderes curativos de grande panóplia de medicinas alternativas, p. ex.

A evolução da sociedade implicou uma noção de direitos individuais que por vezes esquece a noção de dever e de regras, frequentemente exigindo do médico o papel de intermediário burocrático. Por outro lado, por parte de entidades gestoras de cuidados de saúde aumentou a pressão em termos de produtividade. Em diversas circunstâncias e de forma cíclica, tem sido atribuída uma imagem negativa aos médicos, como argumento para a implementação de algumas estratégias de política de saúde.

Todos estes factos contribuem para a construção de uma imagem, de uma expectativa e de uma relação antes da relação. Tais pressupostos estarão presentes, de alguma forma, quando um médico e um paciente se encontram, podendo condicionar o acto médico e a relação terapêutica.

Existem igualmente situações locais específicas que contaminam o momento da consulta. Se um paciente tiver que se sujeitar a uma sala de espera barulhenta, 
sem local para se sentar, organização de serviços deficiente, tempo de espera prolongado, atendimento administrativo inadequado, procedimentos burocratizados, informações imprecisas ou contraditórias, a probabilidade de descontentamento e irritabilidade aumentam consideravelmente.

A existência de muitos pacientes sem Médico de Família, observados em consultas de recurso e não fidelizados a um médico, facilita o doctor-shoppinge a gestão da consulta pelo paciente, o que origina conflito de expectativas.

Dentro do gabinete, outras variáveis podem não ajudar à comunicação. Um paciente e o médico, certamente, sentir-se-ão muito desconfortáveis e inibidos com a falta de privacidade no momento da consulta, com a existência de múltiplas pessoas no consultório, interrupções frequentes ou um gabinete caótico. As diferenças culturais, níveis baixos de literacia ou mesmo línguas maternas diferentes, são outros factores desfavoráveis à compreensão mútua.

\section{CONSEQUÊNCIAS DE UMA RELAÇÃO MÉDICO-PACIENTE DIFÍCIL}

Para um médico, a dificuldade de comunicar ou realizar uma consulta satisfatória pode originar sentimentos muito díspares, que vão da frustração à sensação de incompetência ou de baixa auto-estima. Em função do tipo de paciente, esses sentimentos podem ser de insegurança, irritação, ameaça, rejeição ou mesmo de inutilidade. A sua desvalorização como profissional ou a sensação de desperdício de tempo podem contagiar as consultas subsequentes e toda a sua actividade profissional.

Os pacientes insatisfeitos poderão tornar-se mais exigentes e o médico sentir-se-á menos capaz de responder às suas necessidades, transformando os problemas do sistema de saúde numa frustração interpessoal. ${ }^{2} \mathrm{Um}$ paciente com expectativas insatisfeitas, que se sinta incompreendido, não ouvido nem observado e com queixas ignoradas, transportará o seu mal-estar para outros encontros, num crescendo de insatisfação e de recorrência na vinda à consulta.

Do lado do paciente, a percepção de que o seu médico compreende as prioridades, as expectativas e se preocupa com a sua saúde, está relacionada com melhores resultados, ${ }^{17}$ melhora a sua adequação e o seu acompanhamento, ${ }^{18}$ podendo reduzir o número ou a intensidade das queixas nas visitas seguintes, bem como o medo de uma doença grave. ${ }^{19}$

\section{O QUE FAZER?}

Existem várias estratégias e técnicas susceptíveis de melhorar a gestão de uma «consulta difícil» também aplicáveis e úteis para qualquer outra. A personalidade de alguns médicos será mais facilitadora do que a de outros para intuir alguns caminhos conducentes a uma boa comunicação. No entanto, o modo adequado de comunicar implica uma técnica que se adquire e aperfeiçoa constantemente. Os autores Steinmetz e Tabenkin ${ }^{4}$ (2001) nas entrevistas que fizeram a médicos de família, obtiveram, por ordem decrescente de frequência, várias das qualidades inerentes às melhores formas de lidar com as «consultas ou pacientes difíceis»:

- Empatia

- Escuta sem juízos de valor

- Paciência e tolerância

- Abordagem directa

- Definir os limites de tempo e os temas antecipadamente

- Referenciar a outros profissionais, requisição de exames complementares, contemporizar com medicinas alternativas, recurso a serviços de saúde mental

- Confronto com o paciente

- Recomendação de transferência para outro médico

- Uso do humor

- Envolvimento da família do paciente

- Partilhar algumas experiências pessoais do médico com o paciente

- Ignorar os sentimentos do paciente

Nas já citadas entrevistas de Elder, Ricer e Tobias a 102 «prestigiados médicos de família», ${ }^{5}$ as estratégias sugeridas para lidar com pacientes difíceis foram em muitos aspectos sobreponíveis e, a par da relevância da empatia, da paciência e da tolerância, surgiram como particularmente sublinhadas a necessidade de criar uma ordem de prioridade relativamente às queixas do paciente, ser rigoroso na história e no exame clínicos, usar uma linguagem clara e objectiva, estabelecer regras claras e limites definidos.

Em ambos os estudos é afirmada a existência de um limite para a relação, quando as consultas são persistentemente mal sucedidas, com ultrapassagem de fronteiras sem retorno a partir das quais é aconselhada a 
mudança de médico.

Um incremento da capacidade de escuta levará o paciente a sentir-se com espaço suficiente para si e de que é alvo de um genuíno interesse por parte do clínico. O seu grau de satisfação aumentará. Em cuidados primários, o tempo médio ao fim do qual os médicos interrompem o discurso inicial dos pacientes é de 18 segundos. ${ }^{20}$ Quando as suas exposições não são interrompidas, o tempo de duração média não ultrapassa os 30 segundos. ${ }^{21}$ São doze segundos em que vale a pena investir, pelo que podem significar para a relação, na estruturação da consulta e, provavelmente, na diminuição do «já agora» do fim das consultas.

Os pacientes com perturbação de personalidade necessitarão de uma grande dose de paciência por parte do interlocutor. Em qualquer circunstância, a existência de regras bem definidas e limites claros é facilitadora da relação, melhorando a comunicação e diminuindo a contaminação emocional. Por si só, será estruturante para o paciente. Um médico que não consiga definir regras claras e compreensíveis verá a sua consulta gerida por mais de 1.500 clientes, o que a tornará ingovernável.

Ao invés de fugir a um «doente difícil», a marcação de consultas mais frequentes e o aumento do tempo disponível facilitará a compreensão mútua, o estabelecimento de uma relação de confiança e tenderá a apagar as características «difíceis».

Muitos médicos pensam que as características da sua personalidade e do seu carácter têm um papel no modo como compreendem e lidam com os pacientes difíceis. Por isso, é mais apropriado falar em encontros difíceis e não só em pacientes difíceis. Um paciente difícil para um médico pode não o ser necessariamente para outro. ${ }^{4}$

Mas a noção de cuidados não envolve só os técnicos de saúde. Sabemos que a organização geral dos serviços de saúde, a organização do circuito do utente, o atendimento administrativo e até a organização do espaço, entre outros, têm consequências na satisfação do utente e na relação com o médico. A relação médicopaciente é a ponta do iceberg de um sistema complexo pelo que a melhoria dos resultados dessa parceria não pode ignorar a necessidade de intervenção ao nível de uma equipa mais alargada. Dela fazem parte, entre outros, os dirigentes, os administrativos, os enfer- meiros, os assistentes sociais, os telefonistas, os seguranças, os arquitectos, os responsáveis pela sinalética e os informáticos.

\section{MELHORAR AS CAPACIDADES DE COMUNICAÇÃO}

A maior parte dos autores sugere que a abordagem e gestão de pacientes difíceis deve focar-se na comunicação médico-paciente. O treino do médico em aptidões de comunicação foi previamente associado a um mais apurado reconhecimento de stress emocional mas, desde logo, a maturidade e a experiência pessoais são factores que, implicando uma maior probabilidade de autoconhecimento, facilitam a gestão emocional e a comunicação na consulta. Em casos particulares, a psicoterapia ou a utilização de técnicas de relaxamento pode ser muito útil.

A autoscopia é uma metodologia utilizada no Internato Complementar de Medicina Geral e Familiar, muito útil no reconhecimento de fragilidades e na definição de estratégias para a formação nesta área. O treino pós-graduado relacionado com este tema constitui uma outra via de formação.

O acesso a outros colegas e as relações informais permitem activar o apoio interpares (dentro e fora do local de trabalho) e, possivelmente, é a forma mais comum de descontaminação emocional que fazemos no local de trabalho. Os grupos de apoio, como os Grupos Balint, ${ }^{22}$ são outra forma de suporte interpares que sublinham a importância das emoções e da relação médico-paciente nos resultados clínicos, já que, seguindo a metáfora de Balint, «o médico é o medicamento mais frequentemente prescrito».

O incremento das aptidões comunicacionais por parte do médico, além de aumentar a satisfação profissional e prevenir o burn-out, aumenta a satisfação dos pacientes, diminui a conflituosidade ${ }^{23,24}$ e melhora os resultados em termos de saúde. ${ }^{25}$

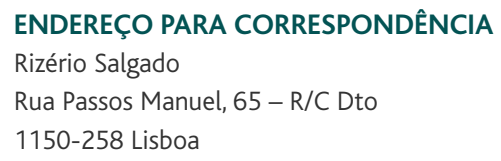

\section{REFERÊNCIAS BIBLIOGRÁFICAS}

1. Jackson JL, Kroenke K. Difficult patient encounters in the ambulatory clinic: clinical predictors and outcomes. Arch Intern Med 1999 May 24; 159 810): 1069-75. 
2. Smith S. Dealing with the difficult patient. Postgrad Med J 1995 Nov; 71 (841): 653-7.

3. Haas JL, Leiser JP, Magill MK, Sanyer ON. Management of the difficult patient. Am Fam Physician 2005 Nov 15; 72 (10): 2063-8.

4. Steinmetz $D$, Tabenkin $H$. The 'difficult patient' as perceived by family physicians. Fam Pract 2001 Oct; 18 (5): 495-500.

5. Elder $N$, Ricer R, tobias $B$. How respected family physicians manage difficult patient encounters. J Am Board Fam Med 2006 Nov-Dec; 19 (6): 533-41.

6. Hahn SR, Thompson KS, Wills TA, Stern V, Budner NS. The difficult doctor-patient relationship: somatization, personality and psychopathology. J Clin Epidemiol 1994 Jun; 47 (6): 647-57.

7. Groves JE. Taking care of the hateful patient. N Engl J Med 1978 Apr 20; 298 (16): 883-7.

8. Kee PC. The difficult patient. Singapore Fam Physician 1995 Apr-Jun. Disponível em; www.cfps.org.sg/sfp/21/212/articles/e212059.html [acedido em 01/05/2008].

9. Hawken SJ. Strategies for dealing with the challenging patient. N Z Fam Phys 2005 Aug; 32 (4): 266-9.

10. Malterud K, Hollnagel $H$, The doctor who cried. Ann Fam Med 2005 JulAug; 3 (4): 348-52.

11. Crutcher JE, Bass MJ. The difficult patient and the troubled physician. J Fam Pract 1980 Nov;11 (6): 933-8.

12. Lin EH, Katon W, Von Korff M, Bush T, Lipscomb P, Russo J, et al. Frustrating patients: physician and patient perspectives among distressed high users of medical services. J Gen Intern Med 1991 Msy-Jun; 6 (3):241-6.

13. Levinson W, Stiles WB, Inui TS, Engle R. Physician frustration in communicating with patients. Med Care 1993 Apr; 31 (4): 285-95.

14. Mathers N, Jones N, Hannay D. Heartsink patients: a study of their general practitioners. Br J Gen Pract 1995 Jun; 45 (395): 293-6.
15. Hull SK, Broquet H. How to manage difficult patient encounters. Fam Pract Manag 2007 Jun; 14 (6): 30-4

16. Groves JE. Taking care of the hateful patient. N Engl J Med 1978 Apr 20; 298 (16): 883-7.

17. Stewart MA. Effective physician-patient communication and health outcomes: a review. CMAJ 1995 May 1; 152 (9): 1423-33.

18. Heisler M, Bouknight RR, Hayward RA, Smith DM, Kerr EA. The relative importance of physician communication, participatory decision making, and patient understanding in diabetes self-management. J Gen Intern Med 2002 Apr; 17 (4): 243-52.

19. Jackson JL, Kroenke K. The effect of unmet expectations among adults presenting with physical symptoms. Ann Intern Med 2001 May 1; 134 (9 Pt 2): 889-97.

20. Beckman HB, Frankel RM. The effect of physician behavior on the collection of data. Ann Intern Med 1984 Nov; 101 (5): 692-6.

21. Nunes JM. Comunicação em contexto clínico. Lisboa: Bayer Health Care; 2007.

22. Balint M. The Doctor, His Patient and the Illness. Edinburgh: Churchill Livingstone, 1963.

23. Virshup BB, Oppenberg AA, Coleman MM. Strategic risk management: reducing malpractice claims through more effective patient-doctor communication. Am J Med Qual 1999 jul-Aug; 14 84): 153-9.

24. Levinson W, Roter DL, Mullooly JP, Dull VT, Frankel RM. Physician-patient communication: the relationship with malpractice claims among primary care physicians and surgeons. JAMA 1997 Feb 19; 277 (7): 553$-9$.

25. Kravitz RL. Measuring patients' expectations and requests. Ann Intern Med 2001 May 1; 134 (9 Pt 2): 881-8.

\footnotetext{
ABSTRACT

In general and family medicine, consultations judged «difficult» occur frequently; they impair health-care delivery and are a source of dissatisfaction both for the doctor and the patient. In this article we analyse the characteristics of the two protagonists that comprise a consultation and of the circumstances that may contribute for the surfacing of a difficult encounter; we also suggest strategies that may improve the communication between doctor and patient and the management of this type of consultations.
}

Keywords: Difficult Patients; Difficult Consultations; Doctor-Patient Communication. 